\title{
A mixed methods study of multiple health behaviors among individuals with stroke
}

\author{
Matthew Plow ${ }^{\text {Corresp., }}{ }^{1}$, Shirley M Moore ${ }^{1}$, Martha Sajatovic ${ }^{2}$, Irene Katzan ${ }^{3}$ \\ ${ }^{1}$ School of Nursing, Case Western Reserve University, Cleveland, Ohio, United States \\ 2 Department of Psychiatry, Neurological and Behavioral Outcomes Center, Case Western Reserve University, Cleveland, Ohio, United States \\ 3 Neurological Institute, Center for Outcomes Research and Evaluation, Cleveland Clinic, Cleveland, Ohio, United States \\ Corresponding Author: Matthew Plow \\ Email address: map208@case.edu
}

Background. Individuals with stroke often have multiple cardiovascular risk factors that necessitate promoting engagement in multiple healthy behaviors. However, observational studies of individuals with stroke have typically focused on promoting a single health behavior. Thus, there is a poor understanding of linkages between healthy behaviors and the circumstances in which factors, such as stroke impairments, may influence a single or multiple health behaviors. Methods. We conducted a mixed methods convergent parallel study of 25 individuals with stroke to examine the relationships between stroke impairments and physical activity, sleep, and nutrition. Our goal was to gain further insight into possible strategies to promote multiple health behaviors among individuals with stroke. This study focused on physical activity, sleep, and nutrition because of their importance in achieving energy balance, maintaining a healthy weight, and reducing cardiovascular risks. Qualitative and quantitative data were collected concurrently, with the former being prioritized over the latter in order to develop a conceptual model of multiple health behaviors. Qualitative and quantitative data were analyzed independently and then were integrated during the inference stage to identify meta-inferences. The 25 individuals with stroke completed closed-ended questionnaires on healthy behaviors and physical function. They also participated in face-to-face focus groups and one-to-one phone interviews. Results. We found statistically significant and moderate correlations between hand function and healthy eating habits $(r=0.45)$, sleep disturbances and limitations in activities of daily living $(r=-0.55)$, BMI and limitations in activities of daily living ( $r=-0.49)$, physical activity and limitations in activities of daily living ( $r=0.41$ ), mobility impairments and BMI $(r=-0.41)$, sleep disturbances and physical activity $(r=-$ $0.48)$, sleep disturbances and BMI $(r=0.48)$, and physical activity and BMI $(r=-0.45)$. We identified five qualitative themes: (1) Impairments: reduced autonomy, (2) Environmental forces: caregivers and information, (3) Re-evaluation: priorities and attributions, (4) 
Resiliency: finding motivation and solutions, and (5) Negative affectivity: stress and selfconsciousness. Three meta-inferences and a conceptual model described circumstances in which factors could influence single or multiple health behaviors. Discussion. This is the first mixed methods study of individuals with stroke to elaborate on relationships between multiple health behaviors, BMI, and physical function. A conceptual model illustrates addressing sleep disturbances, activity limitations, self-image, and emotions to promote multiple health behaviors. We discuss the relevance of the meta-inferences in designing multiple behavior change interventions for individuals with stroke. 
2

5 1) Matthew Plow, PhD (corresponding author): Assistant Professor; Frances Payne Bolton

6 School of Nursing Case Western Reserve University; Frances Payne Bolton School of Nursing,

7 Email: $\underline{\text { map208@case.edu }}$

8

9 2) Shirley M. Moore, PhD, RN: Edward and Louise Mellen Professor of Nursing and Associate

Dean for Research; Frances Payne Bolton School of Nursing, Case Western Reserve University,

Email: Shirely.moore@case.edu

3) Martha Sajatovic, MD: Director, Neurological and Behavioral Outcomes Center,

Department of Psychiatry, Case Western Reserve University School of Medicine, Email:

4) Irene Katzan, MD: Director, Neurological Institute Center for Outcomes Research and 
24

25

26

27

28

\begin{abstract}
Background. Individuals with stroke often have multiple cardiovascular risk factors that necessitate promoting engagement in multiple healthy behaviors. However, observational studies of individuals with stroke have typically focused on promoting a single health behavior. Thus, there is a poor understanding of linkages between healthy behaviors and the circumstances in which factors, such as stroke impairments, may influence a single or multiple health behaviors.
\end{abstract}

Methods. We conducted a mixed methods convergent parallel study of 25 individuals with stroke to examine the relationships between stroke impairments and physical activity, sleep, and nutrition. Our goal was to gain further insight into possible strategies to promote multiple health behaviors among individuals with stroke. This study focused on physical activity, sleep, and nutrition because of their importance in achieving energy balance, maintaining a healthy weight, and reducing cardiovascular risks. Qualitative and quantitative data were collected concurrently, with the former being prioritized over the latter in order to develop a conceptual model of multiple health behaviors. Qualitative and quantitative data were analyzed independently and then were integrated during the inference stage to identify meta-inferences. The 25 individuals with stroke completed closed-ended questionnaires on healthy behaviors and physical function. They also participated in face-to-face focus groups and one-to-one phone interviews.

Results. We found statistically significant and moderate correlations between hand function and healthy eating habits $(r=0.45)$, sleep disturbances and limitations in activities of daily living $(r=-0.55)$, BMI and limitations in activities of daily living $(r=-0.49)$, physical activity and limitations in activities of daily living $(\mathrm{r}=0.41)$, mobility impairments and BMI $(\mathrm{r}=$ $-0.41)$, sleep disturbances and physical activity $(r=-0.48)$, sleep disturbances and BMI $(r=$ 
47 0.48), and physical activity and BMI $(r=-0.45)$. We identified five qualitative themes: (1)

48 Impairments: reduced autonomy, (2) Environmental forces: caregivers and information, (3) Re-

49 evaluation: priorities and attributions, (4) Resiliency: finding motivation and solutions, and (5)

50 Negative affectivity: stress and self-consciousness. Three meta-inferences and a conceptual

51 model described circumstances in which factors could influence single or multiple health

52 behaviors.

Discussion. This is the first mixed methods study of individuals with stroke to elaborate

54 on relationships between multiple health behaviors, BMI, and physical function. A conceptual

55 model illustrates addressing sleep disturbances, activity limitations, self-image, and emotions to

56 promote multiple health behaviors. We discuss the relevance of the meta-inferences in designing

57 multiple behavior change interventions for individuals with stroke. 
Approximately 800,000 people in the United States have a stroke each year (Mozaffarian

70

71

72

73

74

75

et al. 2015). Individuals with stroke are at a significant risk for experiencing a secondary stroke and often have multiple cardiovascular risks factors, such as obesity, hypertension, sleep apnea, and dyslipidemia (Kernan et al. 2014). Reducing these risks requires engagement in multiple health behaviors (Hackam \& Spence 2007). Accordingly, several studies have evaluated behavior change interventions to reduce cardiovascular risk factors in individual with stroke. However, these interventions have largely been ineffective or inconclusive in changing multiple behaviors and reducing cardiovascular risks (Lager et al. 2014). Clearly, fundamental questions remain regarding the best ways to promote multiple health behaviors that reduce risks. For example, it is unknown whether it is more effective to reduce risks by targeting change in multiple behaviors sequentially or simultaneously.

Observational studies of individuals with stroke have typically focused on a single health behavior (Lager et al. 2014). Quantitative studies have identified factors associated with physical activity (Field et al. 2013), medication adherence (O'Carroll et al. 2014), and caregiver interactions (Mackenzie \& Greenwood 2012). Similarly, qualitative studies have described the facilitators for and barriers to engaging in a single health behavior (Chambers et al. 2011; Damush et al. 2007). These studies have documented the negative influence of stroke impairments on behavioral outcomes, such as exercise (Damush et al. 2007; Field et al. 2013) and medication adherence (Chambers et al. 2011; O'Carroll et al. 2014).

However, few studies have addressed questions pertinent to promoting multiple behaviors. For example, rarely are there attempts to understand the linkages between healthy behaviors or how perceptions about one behavior may shape perceptions about another behavior. No studies have examined the circumstances in which stroke impairments may impede the 
92 engagement in single or multiple health behaviors. Furthermore, no studies of individuals with

93 stroke have focused on promoting healthy eating and sleeping habits, two behaviors that may be

94 interrelated with physical activity to reduce cardiovascular risks in individuals with stroke

95 (Grandner et al. 2011; Mozaffarian et al. 2011). Understanding the linkages between stroke

96 impairments and healthy behaviors, such as physical activity, sleep, and nutrition, will be

97 important in reducing cardiovascular risks among strong survivors (Grandner et al. 2011;

98 Mozaffarian et al. 2011).

In order to understand the complexities of engaging in multiple health behaviors while coping with stroke impairments, we conducted a mixed methods study of 25 individuals with stroke. A mixed methods design or the collection and integration of qualitative and quantitative data is particularly relevant to understanding how to promote multiple health behaviors because of the need to determine both the extent and the circumstances in which behaviors are interrelated. Specifically, we implemented a convergent parallel design in which the same 25 individuals with stroke completed closed-ended questionnaires and participated in face-to-face focus groups and one-to-one phone interviews. We decided to focus on physical activity, sleep, and nutrition because of their importance in achieving energy balance, maintaining a healthy weight, and reducing cardiovascular risks (Grandner et al. 2011; Mozaffarian et al. 2011). stroke impairments and physical activity, sleep, and nutrition; 2) to examine the relationships between physical activity, sleep, and nutrition; 3) to gain further insight into possible strategies to promote multiple health behaviors among individuals with stroke. Each purpose was

113 accomplished by employing both qualitative and quantitative methods that were analyzed independently and then were integrated during the inference stage to identify meta-inferences. 
115 The meta-interferences were developed by examining areas of convergence and divergence in

116 the qualitative and quantitative results, with guidance from Noar, Chabot, and Zimmerman's

117 (2008) multiple behavior change framework.

118 Noar et al. (2008) described three approaches to examine multiple health behaviors:

119 behavioral change principles, global health/behavioral categories, and multiple health behaviors.

120 In the behavioral change principles approach, the focus is on examining whether there is a

121 common set of behavioral change principles that can be applied to promote multiple behavioral

122 changes. In the global health/behavioral category approach, the focus is on examining whether

123 there are global health cognitions that predict attitudes towards behavioral categories (e.g.,

124 physical activity) and specific behaviors (e.g., walking), which in turn predict the actual

125 engagement in healthy behaviors. In the multiple behavioral approach, the focus is on examining

126 the linkages between behaviors. We used all three of Noar et al.'s approaches to obtain a

127 comprehensive understanding of engaging in multiple health behaviors.

128 Method

129 Overview

130 A mixed methods convergent parallel fixed design was implemented in which qualitative 131 data was prioritized over quantitative data in order to develop a conceptual model. We aimed to 132 collect different but complementary data to facilitate triangulation and enrich the interpretation 133 of results. Qualitative data was prioritized because of the limited amount of research on multiple

134 health behaviors and the need to develop new hypotheses on how to promote multiple health 135 behaviors. Qualitative and quantitative data were collected in the same order for all participants. 136 All 25 participants first completed the close-ended questionnaires and then participated in one of 137 three face-to-face focus groups, followed by a one-to-one phone interview. Thus, each 
138 participant completed the questionnaires, attended a focus group, and received a follow up phone

139 call. The questionnaires were administered to measure healthy behaviors and physical function.

140 The focus groups were conducted to obtain narratives about engaging in multiple health

141 behaviors. The one-to-one interviews were conducted to help validate interim analyses of the

142 focus groups and to elaborate on the emerging themes. The qualitative data (i.e., focus group and

143 phone interview) and quantitative data (i.e., questionnaires) were analyzed independently and

144 then integrated during the interpretation phase to develop meta-interferences by using Noar et

145 al.'s (2008) framework. An Institutional Review Board at Cleveland Clinic approved the

146 research protocol (IRB \# 11-847). All participants provided written informed consent.

\section{Participants}

148 The study criteria were as follows: a self-report diagnosis of stroke, the ability to

149 communicate over the phone, and a minimum of 18 years of age. We used several strategies to

150 recruit participants for the study. The participants in the first focus group were recruited from a

151 physical therapist-led group exercise class designed for Individuals with stroke. The participants

152 in the second and third focus groups were recruited via flyers posted in the waiting rooms of

153 outpatient neurological and rehabilitation healthcare services.

\section{Questionnaires}

Physical activity was measured using the Godin Leisure-Time Exercise Questionnaire

156 (Godin \& Shephard 1985). Three questions were asked about the frequency of engaging in

157 strenuous (e.g., running and vigorous swimming), moderate (e.g., fast walking, and tennis), and

158 light (e.g., fishing and slow walking) leisure-time exercises for at least 15 minutes. The

159 strenuous, moderate, and light activities were multiplied by 9, 5, and 3, respectively, and then

160 summed to provide a composite score. A higher score indicated a greater level of engagement in 
161 physical activity. The validity and reliability of the Godin Leisure-Time Exercise Questionnaire

162 was validated in several studies and tested in diverse population groups, including those with

163 neurological disabilities. For example, the questionnaire was validated against accelerometers (r

$164=0.53)$ and showed adequate test-retest reliability $(\mathrm{r}=0.77)$ (Godin 2011; Gosney et al. 2007;

165 Noreau et al. 1993; Plow et al. 2012).

166 Sleep disturbance was measured using the Neuro-QOL eight-item short form, version 1

167 (Cella et al. 2012). The questions pertained to the past seven days and were about having trouble

168 getting up in the morning, having trouble stopping thoughts at bedtime, experiencing sleepiness

169 during the day, having trouble falling asleep, and having pain that prevents sleep. The responses

170 to the items ranged from never (1) to always (5) and were summed; higher scores indicated

171 greater problems with sleeping. Neuro-QOL is a set of self-reported, health-related quality of life

172 measures that have been validated (i.e., $\alpha=0.78$; test-retest reliability $\mathrm{ICC}=0.61$ ) in Individuals

173 with stroke (2015; Cella et al. 2012).

174 Healthy nutrition habits were measured using a five-item nutritional survey. The

175 questions asked about the frequency of making healthy food choices, eating five servings of

176 fruits and vegetables a day, limiting fat intake, reading labels, and eating regularly (Nosek et al.

177 2006). The responses to the items were on a scale ranging from never (0), sometimes (1), and

178 often (2) and were summed; higher scores indicated more frequent engagement in healthy

179 nutrition habits. The survey was previously tested in a large study of adults with disabilities

180 (Nosek et al. 2006) and adults with multiple sclerosis (Plow et al. 2012). Its predictive validity

181 (body mass index $r=-0.20)$ and reliability $(\alpha=0.74$; test-retest reliability $r=0.77)$ were found to

182 be adequate. 

2003). The questions pertained to the past two weeks and asked about difficulties in bathing,

185 shopping, walking one block, getting in and out of a car, climbing one flight of stairs, and 186 controlling bladder and bowels. The 16 items were summed and divided into three subscales187 activities of daily living, mobility, and hand function — ranging from 0 to 100; higher scores 188 indicated less pronounced effects of stroke impairments on daily activities. A Rasch analysis was used to construct and validate the 16-item scale based on the physical domain composite of the Stroke Impact Scale. Concurrent validity (i.e., significantly different across disability levels) and reliability ( $\alpha=0.92$ ) were found to be adequate (Duncan et al. 2003; Edwards \& O'Connell 2003).

\section{Questionnaire Analysis}

All data collected from the responses to the questionnaire met the assumption of normality. Thus, Pearson R correlations were used to examine the associations between the responses to the questionnaires and self-reported body mass index (BMI). Statistically significant correlations were shown as two-tailed p-values. A Pearson $\mathrm{R}$ value less than 0.3 was considered small, a value between 0.3 and 0.5 was considered moderate, and a value greater than 0.5 was considered large (Cohen 1988).

\section{Qualitative Interview Procedure}

All interviews were semi-structured (i.e., open-ended questions followed by probes and transitions), audio-recorded, and transcribed verbatim. The focus groups lasted approximately two hours, which were conducted by the first author while a research assistant took notes. The one-to-one interviews lasted approximately 45 minutes, which were conducted over the phone by a trained research assistant. 

motivators for and barriers to engaging in physical activity, nutrition, and sleep. For example, we asked participants whether engaging in physical activity was important and a priority, which types of physical activity they preferred engaging in, and what motivated or hindered and engage in all three behaviors. We then asked participants whether and how they perceived physical activity, nutrition, and sleep to be interrelated and encouraged them to provide examples from their own lives. After each section, the interviewer summarized areas of consensus and disagreement and asked for further input, which helped identify new themes and refine the interview guides. For example, in the second and third focus groups, questions were revised to elaborate on possible themes about spirituality, autonomy, self-image, strategies to manage emotions, and how impairments could hinder multiple behaviors. asked participants if they agreed with the summary and wanted to add anything. Participants were then asked about their social, leisure, occupation, and domestic life roles to establish a rapport with them and to avoid asking irrelevant questions. We then transitioned to a discussion about healthy behaviors by asking the participants to define healthy behaviors and to provide specific examples. Probes were asked to explore global attitudes and perceptions about engagement in healthy behaviors and the perceived relationship of healthy behaviors with recovery and the ability to cope with stroke impairments. Questions were then asked about physical activity, nutrition, and sleep. Compared to the focus groups, more specific questions were asked during the one-to-one interviews to elicit participants' attitudes, knowledge, confidence, outcome expectations, problem-solving strategies, and perceived barriers and 
229 facilitators in their social and physical environments that affected engagement in each of the

230 three behaviors.

\section{Thematic Qualitative Analysis}

232 The thematic analysis was based on recommendations by Elo and Kyngas (2008), while

233

234

235

236

237

238

239

240

241

242

243

244

245

246

247

248

249

250

251

ensuring the trustworthiness of the thematic analysis was based on recommendations by Shenton (2004). The analysis consisted of an inductive-category and theme-development approach to develop the conceptual model. Focus groups and phone interview transcripts were first read multiple times to obtain an overall sense of the data. During the initial reading, the first author and the research assistant performed open coding; i.e., notes were written in the margins of the text. In subsequent readings, similarities and differences between focus group and phone interviews were also noted. Interim analyses identifying similarities and differences between the focus groups and phone interviews helped refine the interview guide and determine when data saturation had occurred.

Notes were compared and discussed to generate descriptive labels that encompassed the data from the focus groups and one-to-one interviews. Overall patterns among the descriptive labels were used to identify clusters to organize the data into themes. Each theme was then operationally defined to facilitate consistent coding of the data. Transcripts were coded using Atlas.ti (Version 7; Scientific Software Development GmbH, Berlin, Germany), which helped generate an audit trail, facilitate the conceptual mapping of categories, and provide flexibility in revising the coding scheme as analyses and discussions occurred.

After the focus group and one-to-one interview transcripts were coded, the first author and the research assistant discussed disagreements and the development of subthemes. Sections of data that were not coded were reviewed, and when necessary, themes and subthemes were 
252 revised to provide a comprehensive description of the data. Once the themes and subthemes were

253 refined and finalized, transcripts and quotes within each theme were re-read to develop the

254 conceptual model. Co-authors who were not involved in the coding of the data examined the

255 appropriateness of each theme and the conceptual model by reviewing exemplar quotes and

256 drawing upon their expertise in nursing, neurology, and psychiatry to help establish content

257 validity.

258 Several steps were taken to help ensure the trustworthiness of the qualitative analysis.

259 Data collection and analysis proceeded iteratively to determine data saturation and identify

260 themes; focus groups were followed by one-to-one interviews (i.e., member checks); an audit

261 trail was generated (i.e., conformability); transcripts were re-read as a whole, and quotes within

262 categories were reviewed and scrutinized on multiple occasions (i.e., dependability); there were

263 frequent debriefing sessions, and disagreements were discussed until consensus was reached

264 (i.e., peer scrutiny); and sections of data that were not coded for the possible inclusion of a new

265 theme (i.e., negative case analysis) were examined.

266 Integrating Qualitative and Quantitative Results

267

At the inference stage, we explored areas of convergence and divergence in the findings

of the qualitative and quantitative analyses to develop and refine meta-inferences and the

conceptual model. Data transformation and typology development were used to integrate the

270

results (Caracelli \& Greene 1993). We first qualitized the quantitative results by writing narrative

271 summaries that described the characteristics of each participant derived from the responses to the

272 questionnaires. We then compared the narrative summaries of participants and generated

273 possible explanations for the similarities and differences in the patterns for engaging in multiple 
274 health behaviors. We further refined the explanations using the calculated means and correlations

275 and then selected the most logical explanations.

276 Qualitizing explanations were then organized in a side-by-side comparison table with

277 qualitative themes and exemplar quotes using Noar et al.'s (2008) framework. Specifically, we

278 organized the qualitizing explanations, qualitative themes, and exemplar quotes based on their

279 relevance in illustrating Noar et al.'s (2008) three approaches for understanding multiple healthy

280 behaviors. We then looked for patterns within each of the approaches to develop the conceptual

281 model and meta-inferences. The conceptual model and meta-inferences were refined using a

282 negative case analysis approach; i.e., we searched for and corrected for any contradictions that

were found between the conceptual model, the meta-inferences, and the narrative summaries of

each participant. Thus, integrating results was an iterative process of comparing and contrasting the qualitative and quantitative data using a theoretical framework. 2006). These strategies included confirming the accuracy of the narrative summaries by a multidisciplinary team of experts and Individuals with stroke (i.e., inside-outside legitimation) by using the qualitative results to elaborate the quantitative associations (i.e., weakness minimization legitimation); analyze the qualitative and quantitative data separately and then develop meta-inferences (i.e., paradigmatic mixing legitimation); use validated questionnaires and strategies to enhance the trustworthiness of the qualitative results (i.e., multiple validities legitimation); and draw upon the existing theoretical literature on multiple healthy behavioral changes (i.e., commensurability legitimation).

\section{Results}


the 25 participants are reported in Table 1. The mean age of the research sample was 64 years; in the number of years since having a stroke (range 1-33 years). Almost two-thirds of the participants were either overweight or obese. Most participants engaged in low amounts of physical activity but indicated that they engaged in some healthy eating habits sometimes or routinely. Sleep disturbances were a common problem. The responses to questions about impairment and healthy behavior are summarized in Table 2, and the Pearson R correlations are reported in Table 3. impairments and healthy behaviors, we found statistically significant and moderate correlations between hand function and healthy eating habits $(\mathrm{r}=0.45)$, sleep disturbances and limitations in activities of daily living $(\mathrm{r}=-0.55)$, BMI and limitations in activities of daily living $(\mathrm{r}=-0.49)$, physical activity and limitations in activities of daily living $(\mathrm{r}=0.41)$, and mobility impairments and BMI $(r=-0.41)$. Regarding the second purpose of the study about examining the relationships between physical activity, sleep, and nutrition, we found statistically significant and moderate correlations between sleep disturbances and physical activity $(r=-0.48)$, sleep disturbances and BMI $(\mathrm{r}=0.48)$, and physical activity and BMI $(\mathrm{r}=-0.45)$. We found nonsignificant and/or small correlations between physical activity and healthy eating habits $(\mathrm{r}=$ $0.33)$ and sleep disturbances and healthy eating habits $(\mathrm{r}=-0.16)$. 

in which participants would set goals to engage in healthy behaviors and would re-assess and

322

323 change goals as needed. This trial and error process was driven by a desire to limit the negative impact of impairments while at the same time being presented with profound barriers that limited the desire and ability to engage in multiple health behaviors. Participants often expressed frustration at being unable or having to put in extra effort to engage in activities as desired. For some participants, this led to greater motivation to engage in multiple health behaviors, whereas for other participants it led to greater amounts of stress. Although some participants believed that engaging in multiple health behaviors was completely under their control, several participants recognized that engaging in multiple health behaviors was influenced by multiple environmental factors that were not always under their control. Healthcare providers, friends and family were described as facilitating and hindering multiple health behaviors. Most participants spent considerable time seeking information about ways to reduce the negative impact of their stroke. We identified the five following overarching themes, with each theme having two to four subthemes: (1) Impairments: reduced autonomy, (2) Environmental forces: caregivers and information, (3) Re-evaluation: priorities and attributions, (4) Resiliency: finding motivation and solutions, and (5) Negative affectivity: stress and self-consciousness. Themes one, two, and three help address the first study purpose to examine the relationships between stroke impairments and healthy behaviors, while categories four and five help address the second study purpose to examine the relationships among healthy behaviors. Table 4 summarizes the categories and subcategories using exemplar quotes.

"Insert Table 4 about here"

(1) Impairments: reduced autonomy. 

described as limiting options, abilities, and confidence to engage in physical activity, nutrition, and sleep. Participants described circumstances in which impairments hindered single or multiple health behaviors. For example, sometimes fatigue was described as only limiting engagement in physical activity, while other times fatigue was described as limiting both physical activity and the ability to eat healthily. Similarly, some participants described how mobility impairments prevented engaging in desired modes of physical activity, such as walking or swimming, due to safety concerns (e.g., fear of falling or drowning). Other participants described how mobility impairments and fatigue made it difficult to cook and/or go grocery shopping, which increased the likelihood of unhealthy food choices (e.g., eating fast food or highly processed food). Pain was described as preventing adequate sleep, engagement in physical activity, and/or the desire to eat healthy foods. altered in desired and undesired ways after the stroke, which facilitated or hindered engagement in multiple health behaviors. For some participants, being unable to work or to accomplish daily tasks and chores resulted in more free time to engage in physical activity and/or they were less tempted to eat unhealthy foods. Participant \#3 said,

"I think it's actually easier now that I'm not working to eat healthy. I think work was than they did because I am just not exposed to those activities, like going to the bar with the guys or having a hot dog after golfing."

Alternatively, others noted being unable to engage in leisure activities, such as golf and 365 gardening, and having fewer daily tasks to accomplish, such as cleaning the house, which made 
366 them more sedentary. Some participants viewed limitations in daily activities and a more

367 sedentary lifestyle as a reason for sleeping difficulties.

368 (2) Environmental forces.

369 Subcategory 1: formal caregivers. Most participants received rehabilitation services and

370 reported occasionally engaging in the prescribed home exercise program. However, participants

371 rarely reported seeing a dietician or a sleep specialist and infrequently had conversations with

372 their physician about engaging in healthy behaviors. Physicians were perceived as being too busy

373 or not knowledgeable in nutrition and physical activity topics, and participants were concerned

374 about discussing sleep problems because of not wanting to be prescribed additional medications.

375 Nonetheless, when a healthcare professional provided advice, participants sometimes described it

376 as a "nudge" to seek further information and/or initiate multiple behavior changes. For example,

377 several participants described trying new diets (e.g., vegetarian) and engaging in different types

378 of physical activity (e.g., yoga and swimming) because their physician recommended it.

379 Alternatively, some participants described engagement in multiple health behaviors as an

380 act of defiance against healthcare advice and recommendations. For example, some participants

381 described being more motivated to engage in physical activity after being told they probably

382 would not see functional improvements after six months. Other participants refused to take

383 prescribed medication and instead ate healthier and engaged in more physical activity.

384 Participant \#6 said,

385 "My doctor told me that no matter what I did, I'd be on medication for all my life. And I

386 refused to accept that and take the medications. As long as I do the exercises and I eat right, my

387 blood pressure will go down.” 
pressure medication increased urination at night, making it difficult to sleep. Participants on

Coumadin had to avoid leafy green vegetables, which was described as a barrier to eating

391

392

393

394 healthily.

Subcategory 2: informal caregivers. Family and friends facilitated healthy behaviors by providing encouragement and tangible support. Several participants were unable to cook or go grocery shopping, which meant they were reliant on others. Not surprisingly, being reliant on others for cooking often influenced dietary habits. Many informal caregivers recognized the importance of physical activity and adequate sleep and would often provide encouragement and tangible support (e.g., transportation or reminders).

Although informal caregivers provided support to engage in healthy behaviors, they also created circumstances that made it more difficult to engage in healthy behaviors. Some participants were more inactive because of an overprotective spouse or child who would limit activities and/or daily chores due to safety concerns. Participant \#13 said, "Well, my kids, they always seem a little surprised at whatever I do. They think I'm supposed to be at home, I think, in a rocking chair so I don't hurt myself. They sometimes do too much for me.” Participants sometimes blamed sleep disturbances on family members who snored or called late at night.

Subcategory 3: seeking information. Most participants were active seekers of information to improve function and to determine why they had a stroke. Participants received health information from a wide variety of sources, such as watching television shows, searching websites, attending support groups, and reading books. Some participants noted that they mainly relied on formal or informal caregivers for health information, which was at times viewed as credible and helpful and was at other times viewed with skepticism and resentment. However, 
411 participants found it challenging to describe how they evaluated the credibility of health

412 information. Some participants trusted most information when it came from an expert or

413 someone they trusted (e.g., a doctor or friend). Other participants were skeptical of most health

414 information, particularly nutrition information, because of contradictory reports or because they

415 were worried about scams.

416 (3) Re-evaluation: priorities and attributions.

417 Subcategory 1: priorities and standards. Reduced autonomy in social roles, increased

418 reliance on formal and informal caregivers, and a wealth of new information to process made

419 participants reflect and re-evaluate priorities and self-image. Impairments made daily chores

420 more challenging and time-consuming, which left less time to prioritize healthy behaviors. Many

421 participants prioritized simple and familiar activities regardless of the known health

422 consequences. In contrast, other participants described re-evaluating priorities to engage in

423 multiple healthy behaviors.

424 Subcategory 2: stroke cause. The decision to prioritize single and multiple healthy

425 behaviors was often attributed to beliefs about what caused the stroke. For example, Participant

426 \#21 said,

“According to what I've read, the lack of exercise sometimes is a factor of diabetes, and it's also a factor of having a stroke. So if I want to avoid that pitfall in the future, it's incumbent upon me to keep myself in the best physical condition that I can, and that's what I'm trying to do [...] by exercising and eating healthy." stroke wasn't really due to my diet. So my diet has not changed [...], but I do try to engage in more activity." 

image and multiple healthy behaviors. Successfully engaging in healthy behaviors improved selfimage and confidence. Achieving health-related goals provided a sense of accomplishment and control in maintaining independence. Some participants described having a more positive selfimage because of increased spirituality and/or having a sense of purpose. Alternatively, participants' unsuccessful attempts at engaging in healthy behaviors decreased confidence and re-enforced a negative self-image.

(4) Resiliency: finding motivation and solutions.

behaviors described having perseverance and persistence. Several participants described having an obstinate approach and refusing to have anything interfere with engagement. Participants successfully engaging in multiple healthy behaviors often had a "just do it" attitude and were confident that nothing could get in their way. Participants determined to engage in multiple healthy behaviors frequently described it as a strategy to cope with stress and impairments. Subcategory 2: strategic environmental planning. Participants described making their home environment conducive to multiple healthy behaviors. Participant \#4 said, access to them. And I - I've been trying to eat those rather than eating junk food, which I've been trying not to keep in the house." their bedroom environment more conducive to sleep. One participant described not wanting their bedroom on the first floor because they wanted to stay physically active by having to climb the 456 stairs. 
458 healthy behaviors were optimistic that maintaining engagement in multiple healthy behaviors would improve health and function. When asked why he was motivated to engage in physical

460 activity, participant \#5 said, "I want to get better. I want to be able to help myself more, you

461 know. I just don't want to be dependent on everybody all the time.” Participant \#21 said,

462 "Getting enough sleep and exercising are the things that contribute to me being able to mentally

463 be stronger and help resist stress or negativity."

(5) Negative affectivity: anxiety and self-consciousness. were described as provoking stress, which in turn exacerbated symptoms, such as fatigue and pain. Some participants worried about another stroke, while others worried about becoming a burden on their families. Such worries resulted in chronic stress or anxiety that could prevent adequate sleep and subsequent engagement in physical activity and nutrition-related behaviors. Several participants stated that increased stress and/or inadequate sleep exacerbated symptom severity and resulted in having "good days and bad days", which influenced multiple healthy 472 behaviors. and embarrassment, were described as influencing healthy behaviors in social and community settings. Some participants described being uncomfortable exercising at community centers. Participants also described limiting social and leisure physical activities because of difficulties

477 walking and speaking. Participants with upper-extremity mobility impairments described

478 reluctantly ordering unhealthy fried finger foods at restaurants to avoid the embarrassment and

479 difficulties associated with eating healthier foods that required a fork, such as a salad. Body- 
480 related, self-conscious emotions and limiting options also propagated a negative self-image.

481 Participant \#12 said,

482 'It's just this and the effect the stroke had on me, and it's made me very sensitive [about 483 my body]. You know, I find myself questioning myself on everything I do - whether I'm doing 484 the right thing or whether I'm doing it well enough."

485 Integrated Results: Meta-Inferences and the Conceptual Model

486 We identified three meta-inferences: reciprocal determinism correspondence,

487 circumstantial linkages, and the sleep disturbance ripple effect. The conceptual model shown in 488 Figure 1 elaborates on the relationship between the thematic categories and quantitative data, 489 490 491 which indicated factors that promote or hinder the engagement in multiple healthy behaviors.

"Figure 1 and Table 5 about here"

\section{Meta-inference \#1: Reciprocal determinism correspondence}

Behavioral change principles approach. Reciprocal determinism refers to Bandura's principle of how participants described dynamic interactions between person, behavior, and the environment (Bandura 1986). Correspondence refers to participants providing examples of reciprocal deterministic relationships that were relevant to multiple health behaviors and specific reciprocal deterministic relationships that were relevant to a single healthy behavior. As interacting with environmental factors, such as inadequate social support, prompted changes in priorities and standards, which often resulted in frustration and stress that decreased motivation, confidence, and/or the ability to engage in multiple healthy behaviors. Alternatively, some participants described that a specific impairment, such as fatigue, would interact with a specific 
503 healthy behavior, such as physical activity. Correspondence was also supported by quantitative 504 results. For example, activities of daily living — an indicator of multiple impairments or a more

505 severe impairment—were inversely associated with multiple health behaviors, whereas problems

506 with hand function - a specific impairment — were inversely associated with a single healthy

507 behavior; i.e., nutrition.

508 Meta-inference \#2: Circumstantial linkages

509 Global health/behavioral category approach. The term circumstantial linkages refers to

510 relationships between behaviors and whether a factor, such as a global cognition or a

511 psychological disposition, such as resilience or negative affectivity, influenced single or multiple

512 behaviors depending on the circumstances (e.g., environment, personality traits, and cognitions)

513 of each individual. Although the participants described many scenarios that indicated possible

514 links between global cognitions, behavioral categories, and specific behaviors (as suggested by

515 Noar et al., 2008), these descriptions were not always consistent. For example, in the qualitative

516 interviews, fatigue and pain were sometimes described as hindering single or multiple healthy

517 behaviors, depending on environmental circumstances, such as the availability of tangible social

518 support. Some participants described prioritizing one healthy behavior over another and

519 rationalizing that one healthy behavior was more important than another healthy behavior. Other

520 participants noted the importance of prioritizing multiple healthy behaviors, expressed

521 confidence that the engagement in multiple healthy behaviors was completely under their

522 control, and described personal traits, such as resiliency, that facilitated multiple behaviors.

523 Circumstantial linkages were also supported by the quantitative data, specifically the low-

524 to-moderate correlations between different behaviors. For example, we found a small, non-

525 significant correlation between physical activity and nutrition. Nonetheless, some participants 
526 described the importance of routinely engaging in both physical activity and eating healthily,

527 whereas other participants described prioritizing either physical activity or eating healthily. If the

528 participants were motivated to engage in both physical activity and eating healthily regardless of

529 individual circumstances, it is likely that higher correlations would have been found.

530 Meta-inference 3: Sleep-disturbance ripple effect

531 Multiple behavioral approach. The sleep disturbance ripple effect refers to the influence

532 of inadequate sleep on the factors that hinder the engagement in multiple behaviors. We found

533 sleep disturbances to have moderate correlations with physical activity, BMI, and the ability to

534 perform activities of daily living. In the qualitative data, sleep disturbances were described as

535 increasing stress and fatigue, exacerbating impairments (e.g., pain, mobility, and fatigue), and

536 decreasing the motivation to engage in multiple healthy behaviors, such as avoiding unhealthy

537 temptations, cooking a healthy meal, and engaging in physical activity. Thus, both the qualitative

538 and the quantitative data supported the importance of obtaining adequate sleep for engaging in

539 multiple health behaviors.

540 Discussion

541 The science of how to promote multiple health behaviors is in its formative stage, which

542 has considerable consequences for individuals with stroke. Targeting multiple behaviors has an

$54380 \%$ cumulative risk reduction in preventing secondary strokes (Hackam \& Spence 2007). Thus,

544 there is a need to promote multiple health behaviors among individuals with stroke. We have

545 conducted a novel mixed methods study to elaborate on the relationships between multiple

546 healthy behaviors and to identify factors that facilitate and/or hinder multiple health behaviors.

547 Our mixed methods results advance existing research by identifying meta-inferences that

548 describe how stroke impairments may be barriers to single or multiple health behaviors. We also 
549 developed a conceptual model depicting possible mediators that influence the relationship

550 between stroke impairments and multiple health behaviors.

551 Addressing Purpose 1: Stroke Impairments and Multiple Healthy Behaviors

552 Several participants described engaging in multiple healthy behaviors as a strategy to

553 cope with stroke impairments. Quantitative studies of single health behaviors among individuals

554 with stroke have also documented that emotional coping strategies and mood (Visser et al. 2014),

555 physical activity (Chen \& Rimmer 2011), sleep (Cavalcanti et al. 2013), nutrition (Serra et al.

556 2014), and environmental factors (Alguren et al. 2012) influenced the process of adjusting to

557 stroke impairments. Similarly, in a systematic review of qualitative studies (Sarre et al. 2014),

558 resiliency, informal and formal caregivers, and engaging in healthy behaviors were described as

559 influencing adjustment after a stroke. Thus, promoting engagement in multiple health behaviors

560 may be a strategy to help individuals with stroke cope with their impairments and improve their

561 quality of life.

562 However, the challenge in promoting multiple healthy behaviors is addressing reciprocal

563 relationship; i.e., impairments that make it difficult to engage in healthy behaviors. Future

564 research should determine whether the negative impact of impairments on multiple health

565 behaviors may be meditated by environmental factors (e.g., caregivers) and participants'

566 characteristics (e.g., resiliency, mood, self-image, optimism, and perceptions about the causes of

567 the stroke). Positive psychology constructs, such as resilience and optimism, are considered to be

568 modifiable characteristics that may help facilitate the adjustment to a chronic disabling condition

569 and the engagement in multiple health behaviors (Martz \& Livneh 2015).

570 Addressing Purpose 2: Linkages among Healthy Behaviors 
The results of this study showed that the linkages between healthy behaviors might be

572 dependent on the characteristics of the participant. This finding is consistent with a recent

573 research study of participants who were undergoing a rehabilitation program. The results of that

574 quantitative study indicated that the relationship between physical activity and nutrition was

575 mediated by habit strength and transfer cognition (Fleig et al. 2014). Research has also indicated

576 that promoting the engagement in one behavior (e.g., physical activity) might promote the

577 engagement in another behavior (e.g., reducing cigarette craving) (Haasova et al. 2013; Noar et

578 al. 2008). Mixed methods studies of multiple health behaviors have mainly focused on weight

579 management among non-disabled children and adults. Such studies have documented

580 circumstances in which people can increase physical activity levels and/or develop healthy eating

581 habits (Abildso et al. 2010; James et al. 2016; Sliwa et al. 2014).

582 Consistent with the existing research, we found that sleep had moderate correlations with

583 physical activity, BMI, and the ability to perform activities in daily living (Bakken et al. 2012;

584 Cavalcanti et al. 2013; Grandner et al. 2011). The participants described that sleep disturbances

585 resulted in fatigue and negative emotions, which decreased their motivation to engage in multiple

586 healthy behaviors. A mixed methods study of sleep habits among patients with traumatic brain

587 injury showed that inadequate sleep had a major impact on health outcomes and reduced

588 adherence to rehabilitation activities (De La Rue-Evans et al. 2013). Future studies of individuals

589 with stroke should examine whether sleep disturbances need to be addressed before multiple

590 behavior changes can occur.

591 Addressing Purpose 3: Developing Multiple Behavior Change Interventions

592 The results indicated that future research should examine the efficacy of multiple

593 behavioral change interventions that focus on increasing resiliency and self-confidence and that 
594 address negative emotions and sleep disturbances. Such multiple behavioral change interventions

595 might include education about identifying creditable sources of information, re-arranging the 596 social and physical environment, promoting social support, exploring participants' perceptions

597 about what caused their stroke, and providing examples of how engaging in multiple healthy 598 behaviors may help reduce the need for medication. Multiple behavioral change interventions 599 may also need to target participants who rationalize the prioritization of one particular healthy 600 behavior over another.

601 In a recent review of qualitative studies, Lawrence et al. (2016) reported that individuals 602 with stroke generally perceived that participating in behavior change interventions was 603 beneficial. They described feeling supported and that they acquired new knowledge and gained 604 increased confidence to engage in healthy behaviors. These findings were consistent with our 605 recommendation to identify creditable sources of information and increase confidence and social 606 support in the design of multiple behavioral change interventions. Therefore, existing 607 interventions could be adapted to promote multiple behavioral changes in individuals with 608 stroke.

609 Self-management interventions focus on increasing confidence and teaching skills, such 610 as problem-solving and resource utilization, which may be relevant to promoting engagement in 611 single or multiple behaviors to manage single or multiple impairments (Lo et al. 2013; Lorig \& 612 Holman 2003; Parke et al. 2015). However, the efficacy and theoretical underpinnings of these 613 interventions to support multiple behavioral changes are unclear. The results of a recent mixed 614 methods study of a tailored self-management program showed promise in promoting patient 615 activation, exercise, and healthy eating (Montgomery et al. 2015). Future studies should 
616 incorporate objective and self-report measures of healthy behaviors to determine whether self-

617 management interventions can promote multiple health behaviors.

618 Application to Noar et al. 's framework. We identified three meta-inferences that were

619 consistent with Noar et al.'s (2008) three approaches to understanding and promoting multiple

620 behavioral changes. Regarding the use of the behavioral change principle approach, promoting

621 multiple health behaviors simultaneously may require identifying and addressing reciprocal

622 deterministic relationships that correspond with multiple health behaviors. Similarly, identifying

623 and addressing the relationships that correspond with a single health behavior may be a strategy

624 to promote multiple behavior changes sequentially. Regarding the global health/behavioral

625 category approach, targeting and improving global cognitions and dispositions, such as resiliency

626 and optimism, may help promote multiple health behaviors. However, further research is needed

627 to determine the circumstances in which global cognition could be addressed. Although targeting

628 global cognitions has the allure of promoting multiple behaviors simultaneously, they may be

629 harder to change than cognitions about particular behaviors (Magidson et al. 2014). Furthermore,

630 successfully changing global cognitions may not always influence behaviors in the same way in

631 all participants (Blackie et al. 2014). Regarding the multiple behavioral approach, we found that

632 it might be important to target sleep disturbances to promote multiple behavior changes.

633 Limitations

634 The limitations of this study include the small sample size, the cross-sectional design, and

635 the generalizability of the results. Because little is known about the linkages between multiple

636 healthy behaviors, which makes it difficult to generate a-priori hypotheses, we decided to

637 prioritize qualitative data over quantitative data. Therefore, although the sample size was

638 sufficient for the qualitative analysis, it limited the findings of the quantitative analysis. The 
639 small sample size precluded the ability to conduct a valid multiple regression analysis, while the 640 cross-sectional design precluded the ability able to make inferences about causal relationships.

641 Furthermore, the measures of physical activity and nutrition were not specifically validated in

642 individuals with stroke, and they may have influenced correlations in unknown ways. The 643 generalizability of the study is limited because of the nature of the qualitative analysis and the

644 small sample size used in the quantitative analysis. We did not include adults with severe aphasia 645 or cognitive impairments. Thus, results may not be relevant to individuals with stroke with these 646 severe impairments. In addition, the recruitment of the first focus group was potentially biased 647 because it was limited to the participants in a physical therapy group exercise class. Finally, the 648 incorporation of the additional measures of personality and cognition could have helped promote 649 greater triangulation of the findings. A sequential mixed design that analyzed qualitative data 650 first would have enabled the selection of additional questionnaires to facilitate triangulation.

\section{Conclusion}

652 Coping skills, social support, and type and extent of impairments are just some of the complexities that may influence engagement in multiple healthy behaviors among adults with

654

655

656

657

658

659

660

661 disabling conditions. The use of mixed methods can help examine such complexities from different angles to offer a more holistic prospective of how to promote engagement in multiple health behaviors. Researchers in rehabilitation science and public health have noted the need to use mixed methods to understand the complex biopsychosocial interactions that result in participation restrictions for engaging in healthy behaviors (Brownson et al. 2014; Kroll 2011).

Regarding the question about whether multiple behavior change interventions should target behaviors sequentially and/or simultaneously, the answer may depend on the characteristics of the participants. We found examples of facilitators and barriers that were 
662 described as being relevant to a single behavior and in other circumstances as being relevant to 663 multiple healthy behaviors. Therefore, the extent to which a particular facilitator and/or barrier 664 influences multiple behaviors may depend on cognition and environmental circumstances.

665 Examining the circumstances in which factors influence single or multiple behaviors and 666 understanding when it is possible to change factors that promote multiple behavior changes will 667 be vital in developing multiple behavior change interventions among Individuals with stroke.

668 Further mixed methods research is needed to identify the circumstances that influence single or 669 multiple behaviors and the particular factors that may influence multiple behaviors regardless of 670 the circumstances.

671

672

673

674

675

676

677

678

679

680

681

682

683

684

685

686

687

688

689

690

691

692

693

694

695

696 
697

698

699

700

701

702

703

704

705

706

707

708

709

710

711

712

713

714

715

716

717

718

\section{References}

2015. Neuro-QoL Technical Report. Available at http://www.neuroqol.org/Resources/Resources\%20documents/NeuroQoL\%20Manual Technical\%20Report\%20v2 24Mar2015.pdf (accessed 10/30/2015.

Abildso C, Zizzi S, Gilleland D, Thomas J, and Bonner D. 2010. A Mixed Methods Evaluation of a 12-Week Insurance-Sponsored Weight Management Program Incorporating Cognitive-Behavioral Counseling. Journal of Mixed Methods Research 4:278-294. $10.1177 / 1558689810376949$

Alguren B, Fridlund B, Cieza A, Sunnerhagen KS, and Christensson L. 2012. Factors associated with health-related quality of life after stroke: A 1-year prospective cohort study. Neurorehabilitation and Neural Repair 26:266-274. 10.1177/1545968311414204

Bakken LN, Kim HS, Finset A, and Lerdal A. 2012. Stroke patients' functions in personal activities of daily living in relation to sleep and socio-demographic and clinical variables in the acute phase after first-time stroke and at six months of follow-up. Journal of Clinical Nursing 21:1886-1895. 10.1111/j.1365-2702.2011.04014.x

Bandura A. 1986. Social Foundations of Thought and Action: A Social Cognitive Theory. Englewood Cliffs (NJ): Prentice-Hall.

Blackie LER, Roepke AM, Forgeard MJC, Jayawickreme E, and Fleeson W. 2014. Act Well to Be Well: The Promise of Changing Personality States to Promote Well-Being. The Wiley Blackwell Handbook of Positive Psychological Interventions: John Wiley \& Sons, Ltd, 462-474. 
719 Brownson RC, Diez Roux AV, and Swartz K. 2014. Commentary: Generating rigorous evidence 720 for public health: the need for new thinking to improve research and practice. Annual

721

722

723

724

725

726

727

728

729

730

731

732

733

734

735

736

737

738

739

740 Review of Public Health 35:1-7. 10.1146/annurev-publhealth-112613-011646

Caracelli VJ, and Greene JC. 1993. Data Analysis Strategies for Mixed-Method Evaluation Designs. Educational Evaluation and Policy Analysis 15:195-207. $10.3102 / 01623737015002195$

Cavalcanti PR, Campos TF, and Araüjo JF. 2013. Circadian and homeostatic changes of sleepwake and quality of life in stroke: Implications for neurorehabilitation. NeuroRehabilitation 32:337-343. 10.3233/NRE-130853

Cella D, Lai JS, Nowinski CJ, Victorson D, Peterman A, Miller D, Bethoux F, Heinemann A, Rubin S, Cavazos JE, Reder AT, Sufit R, Simuni T, Holmes GL, Siderowf A, Wojna V, Bode R, McKinney N, Podrabsky T, Wortman K, Choi S, Gershon R, Rothrock N, and Moy C. 2012. Neuro-QOL: Brief measures of health-related quality of life for clinical research in neurology. Neurology 78:1860-1867. 10.1212/WNL.0b013e318258f744

Chambers JA, O'Carroll RE, Hamilton B, Whittaker J, Johnston M, Sudlow C, and Dennis M. 2011. Adherence to medication in Individuals with stroke: A qualitative comparison of low and high adherers. British Journal of Health Psychology 16:592-609. 10.1348/20448287.002000

Chen MD, and Rimmer JH. 2011. Effects of exercise on quality of life in Individuals with stroke: A meta-analysis. Stroke 42:832-837.

Cohen J. 1988. Statistical Power Analysis for the Behavioral Sciences. Hillsdale, N.J.: L. Erlbaum Associates. 
741 Damush TM, Plue L, Bakas T, Schmid A, and Williams LS. 2007. Barriers and facilitators to 742 exercise among Individuals with stroke. Rehabilitation Nursing 32:253-260, 262.

743

744

745

746

747

748

749

750

751

752

753

754

755

756

757

758

759

760

761

De La Rue-Evans L, Nesbitt K, and Oka RK. 2013. Sleep Hygiene Program Implementation in Patients with Traumatic Brain Injury. Rehabilitation Nursing 38:2-10. 10.1002/rnj.66

Duncan PW, Lai SM, Bode RK, Perera S, and DeRosa J. 2003. Stroke Impact Scale-16: A brief assessment of physical function. Neurology 60:291-296.

Edwards B, and O'Connell B. 2003. Internal consistency and validity of the Stroke Impact Scale 2.0 (SIS 2.0) and SIS-16 in an Australian sample. Quality of Life Research 12:1127-1135.

Elo S, and Kyngas H. 2008. The qualitative content analysis process. Journal of Advanced Nursing 62:107-115. 10.1111/j.1365-2648.2007.04569.x

Field MJ, Gebruers N, Shanmuga Sundaram T, Nicholson S, and Mead G. 2013. Physical activity after stroke: A systematic review and meta-analysis. International Scholarly Research Notices Stroke 2013.

Fleig L, Kerschreiter R, Schwarzer R, Pomp S, and Lippke S. 2014. 'Sticking to a healthy diet is easier for me when I exercise regularly': Cognitive transfer between physical exercise and healthy nutrition. Psychology \& Health 29:1361-1372.

Godin G. 2011. The Godin-Shephard leisure-time physical activity questionnaire. The Health \& Fitness Journal of Canada 4:18-22.

Godin G, and Shephard RJ. 1985. A simple method to assess exercise behavior in the community. Canadian Journal of Applied Sport Sciences Journal Canadien des Sciences Appliquees Au Sport 10:141-146. 
762 Gosney JL, Scott JA, Snook EM, and Motl RW. 2007. Physical activity and multiple sclerosis -

763 Validity of self-report and objective measures. Family and Community Health 30:144$764 \quad 150$.

765 Grandner MA, Patel NP, Perlis ML, Gehrman PR, Xie D, Sha D, Pigeon WR, Teff K, Weaver T, 766 and Gooneratne NS. 2011. Obesity, diabetes, and exercise associated with sleep-related 767 complaints in the American population. Journal of Public Health 19:463-474.

768 Haasova M, Warren FC, Ussher M, Janse Van Rensburg K, Faulkner G, Cropley M, ByronDaniel J, Everson-Hock ES, Oh H, and Taylor AH. 2013. The acute effects of physical activity on cigarette cravings: systematic review and meta-analysis with individual participant data. Addiction 108:26-37. 10.1111/j.1360-0443.2012.04034.x

772 Hackam DG, and Spence JD. 2007. Combining multiple approaches for the secondary prevention of vascular events after stroke: A quantitative modeling study. Stroke 38:1881-1885. 10.1161/STROKEAHA.106.475525

James DCS, Wirth CK, Harville C, and Efunbumi O. 2016. Weight-loss strategies used by baby boomer men: a mixed methods approach. Journal of Human Nutrition and Dietetics 29:217-224. 10.1111/jhn.12305

778

779

780

781

782

783

Kernan WN, Ovbiagele B, Black HR, Bravata DM, Chimowitz MI, Ezekowitz MD, Fang MC, Fisher M, Furie KL, Heck DV, Johnston SC, Kasner SE, Kittner SJ, Mitchell PH, Rich MW, Richardson D, Schwamm LH, Wilson JA, American Heart Association Stroke Council CoC, Stroke Nursing CoCC, and Council on Peripheral Vascular D. 2014. Guidelines for the prevention of stroke in patients with stroke and transient ischemic attack: a guideline for healthcare professionals from the American Heart 
784

785

786

787

788

789

790

791

792

793

794

795

796

797

798

799

800

801

802

803

804

805

806

Association/American Stroke Association. Stroke 45:2160-2236.

10.1161/STR.0000000000000024

Kroll T. 2011. Designing mixed methods studies in health-related research with people with disabilities. International Journal of Multiple Research Approaches 5:64-75. 10.5172/mra.2011.5.1.64

Lager KE, Mistri AK, Khunti K, Haunton VJ, Sett AK, and Wilson AD. 2014. Interventions for improving modifiable risk factor control in the secondary prevention of stroke. Cochrane Database of Systematic Reviews 5:CD009103. 10.1002/14651858.CD009103.pub2

Lawrence M, Pringle J, Kerr S, and Booth J. 2016. Individuals with stroke' and family members' perspectives of multimodal lifestyle interventions for secondary prevention of stroke and transient ischemic attack: a qualitative review and meta-aggregation. Disability and Rehabilitation 38:11-21. 10.3109/09638288.2015.1031831

Lo SHS, Chang AM, Chau JPC, and Gardner G. 2013. Theory-based self-management programs for promoting recovery in community-dwelling Individuals with stroke: A systematic review. JBI Database of Systematic Reviews and Implementation Reports 11:157-215.

Lorig KR, and Holman H. 2003. Self-management education: History, definition, outcomes, and mechanisms. Annals of Behavioral Medicine 26:1-7.

Mackenzie A, and Greenwood N. 2012. Positive experiences of caregiving in stroke: A systematic review. Disability and Rehabilitation 34:1413-1422. doi:10.3109/09638288.2011.650307

Magidson JF, Roberts BW, Collado-Rodriguez A, and Lejuez CW. 2014. Theory-driven intervention for changing personality: Expectancy value theory, behavioral activation, and conscientiousness. Developmental Psychology 50:1442-1450. 10.1037/a0030583 
807 Martz E, and Livneh H. 2015. Psychosocial Adaptation to Disability Within the Context of 808 Positive Psychology: Findings from the Literature. Journal of Occupational 809 Rehabilitation:1-9.

810 Montgomery P, Jermyn D, Bailey P, Nangia P, Egan M, and Mossey S. 2015. Community 811 reintegration of Individuals with stroke: the effect of a community navigation 812 intervention. Journal of Advanced Nursing 71:214-225.

813 Mozaffarian D, Benjamin EJ, Go AS, Arnett DK, Blaha MJ, Cushman M, de Ferranti S, Despres JP, Fullerton HJ, Howard VJ, Huffman MD, Judd SE, Kissela BM, Lackland DT, Lichtman JH, Lisabeth LD, Liu S, Mackey RH, Matchar DB, McGuire DK, Mohler ER, 3rd, Moy CS, Muntner P, Mussolino ME, Nasir K, Neumar RW, Nichol G, Palaniappan L, Pandey DK, Reeves MJ, Rodriguez CJ, Sorlie PD, Stein J, Towfighi A, Turan TN, Virani SS, Willey JZ, Woo D, Yeh RW, Turner MB, American Heart Association Statistics C, and Stroke Statistics S. 2015. Heart disease and stroke statistics--2015 update: a report from the American Heart Association. Circulation 131:e29-322.

\subsection{1/CIR.0000000000000152}

Mozaffarian D, Hao T, Rimm EB, Willett WC, and Hu FB. 2011. Changes in diet and lifestyle and long-term weight gain in women and men. New England Journal of Medicine

825 Noar SM, Chabot M, and Zimmerman RS. 2008. Applying health behavior theory to multiple behavior change: Considerations and approaches. Preventive Medicine 46:275-280. 10.1016/j.ypmed.2007.08.001 
828 Noreau L, Shephard RJ, Simard C, Pare G, and Pomerleau P. 1993. Relationship of impairment

829 and functional ability to habitual activity and fitness following spinal cord injury.

830 International Journal of Rehabilitation Research 16:265-275.

831 Nosek MA, Hughes RB, Robinson-Whelen S, Taylor HB, and Howland CA. 2006. Physical

832

833

834

835

836

837

838

839

840

841

842

843

844

845

846

847

848

849

850

activity and nutritional behaviors of women with physical disabilities: physical, psychological, social, and environmental influences. Womens Health Issues 16:323-333. 10.1016/j.whi.2006.08.002

O’Carroll RE, Chambers JA, Dennis M, Sudlow C, and Johnston M. 2014. Improving medication adherence in Individuals with stroke: Mediators and moderators of treatment effects. Health Psychology 33:1241-1250. 10.1037/hea0000082

Onwuegbuzie AJ, and Johnson RB. 2006. The validity issue in mixed research. Research in the Schools 13:48-63.

Parke HL, Epiphaniou E, Pearce G, Taylor SJ, Sheikh A, Griffiths CJ, Greenhalgh T, and Pinnock H. 2015. Self-Management Support Interventions for Individuals with stroke: A Systematic Meta-Review. PLoS One 10:e0131448. 10.1371/journal.pone.0131448

Plow M, Finlayson M, and Cho C. 2012. Correlates of nutritional behavior in individuals with multiple sclerosis. Disability and Health Journal 5:284-291.

Sarre S, Redlich C, Tinker A, Sadler E, Bhalla A, and McKevitt C. 2014. A systematic review of qualitative studies on adjusting after stroke: Lessons for the study of resilience. Disability and Rehabilitation 36:716-726. 10.3109/09638288.2013.814724

Serra MC, Hafer-Macko CE, Ivey FM, Macko RF, and Ryan AS. 2014. Impact of Serum Nutritional Status on Physical Function in African American and Caucasian Individuals with stroke. Stroke research and treatment 2014. 
851 Shenton AK. 2004. Strategies for ensuring trustworthiness in qualitative research projects.

852 Education for information 22:63-75.

853 Sliwa SA, Sharma S, Dietz WH, Dolan PR, Nelson ME, and Newman MB. 2014. Healthy Kids

854 Out of School: Using Mixed Methods to Develop Principles for Promoting Healthy

855 Eating and Physical Activity in Out-of-School Settings in the United States. Preventing

856 chronic disease 11:E227. 10.5888/pcd11.140207

857 Visser MM, Aben L, Heijenbrok-Kal MH, Busschbach JJ, and Ribbers GM. 2014. The relative

858

859

860

861

862

863

864

865

866

867

868

869

870

871

872

873

874

875

876

877

878

879

880

881

882

883 
884 Table 1: Characteristics of the participants $(n=25)$

\begin{tabular}{ll}
\hline & Mean (range) \\
\hline Age (years) & $64.12(46-89)$ \\
Years since first stroke & $5.68(1-33)$ \\
\hline Gender & NFrequency count (\%) \\
Female & $12(48)$ \\
Racial minority & $5(20)$ \\
Education (>15 years) & $8(57)$ \\
Living with someone else & $19(76)$ \\
Living with spouse & $16(64)$ \\
Body mass index & \\
Underweight & $1(4)$ \\
Normal & $6(24)$ \\
Overweight & $10(40)$ \\
Obese & $8(32)$ \\
Cane use* & \\
Never & 11 \\
Sometimes & 5 \\
Always & 7 \\
Walker use & 17 \\
Never & 5 \\
Sometimes & 2 \\
Always & 14 \\
Wheelchair use & 6 \\
Never & 4 \\
Sometimes & \\
Always & \\
\hline Key: & \\
\hline & \\
\hline &
\end{tabular}

Key: *Participant could report using multiple mobility aids.

886

887

888

889

890 
Table 2: Mean, standard deviation, and range of healthy behaviors and physical function

892 questionnaires

\begin{tabular}{lll}
\hline Variable & M (SD) & Range \\
\hline Healthy eating habits & $7.96(1.49)$ & $5.00-10.00$ \\
Physical activity & $33.20(27.54)$ & $0.00-100.00$ \\
Sleep disturbances & $15.20(5.26)$ & $8.00-27.00$ \\
ADL limitations & $78.02(16.37)$ & $37.50-100.00$ \\
Mobility & $72.40(19.09)$ & $39.29-100.00$ \\
Hand function & $49.00(35.71)$ & $0.00-100$
\end{tabular}

893

894

895

896

897

898

899

900

901

902

903

904

905

906

907

908

909

910

911

Note. Sleep disturbance: Neuro-QOL eight-item short form; Physical activity: Godin Leisure-

Time Exercise Questionnaire; Healthy eating habits: five-item questionnaire on healthy eating habits; ADL limitations: Activities of Daily Living subscale of Stroke Impact Scale-16;

Mobility: subscale of Stroke Impact Scale-16; Hand function: subscale of Stroke Impact Scale16.

8

9

(1)


912 Table 3: Pearson correlations between healthy behaviors and health-related quality of life

913 questionnaires

\begin{tabular}{lcccccc}
\hline Variables & BMI & $\begin{array}{c}\text { Physical } \\
\text { activity }\end{array}$ & Sleep & $\begin{array}{c}\text { Healthy } \\
\text { eating habits }\end{array}$ & $\begin{array}{c}\text { ADL } \\
\text { limitations }\end{array}$ & Mobility \\
\hline Physical activity & $-0.45^{*}$ & & & & & \\
Sleep disturbances & $0.48^{*}$ & $-0.48^{*}$ & & & & \\
Healthy eating habits & -0.10 & 0.33 & -0.16 & & & \\
ADL limitations & $-0.49^{*}$ & $0.41^{*}$ & $-0.55^{* *}$ & $0.42^{*}$ & & \\
Mobility & $-0.41^{*}$ & 0.39 & -0.19 & 0.33 & $0.78^{* *}$ & \\
Hand function & 0.05 & 0.20 & -0.20 & $0.45^{*}$ & $0.70^{* *}$ & $0.71^{* *}$ \\
\hline
\end{tabular}

914 Note. $*$ Correlation is significant at the 0.05 level (two-tailed). ${ }^{*}$ Correlation is significant at the

9150.01 level (two-tailed). BMI: body mass index (self-report); Sleep disturbance: Neuro-QOL

916 eight-item short form; Physical activity: Godin Leisure-Time Exercise Questionnaire; Healthy

917 eating habits: five-item questionnaire on nutrition-related behaviors; ADL limitations:

918 Activities of Daily Living subscale of Stroke Impact Scale-16; Mobility: subscale of Stroke

919 Impact Scale-16; Hand function: subscale of Stroke Impact Scale-16.

920

921

922

923

924

925

926

927

928

929

930

931

932

933

934

935

936

937

938

939

940 
941 Table 4: Summary of themes and subthemes

\begin{tabular}{|c|c|c|}
\hline Themes & Subthemes & Examples \\
\hline \multirow[t]{3}{*}{$\begin{array}{l}\text { Impairments: } \\
\text { reduced } \\
\text { autonomy }\end{array}$} & $\begin{array}{l}\text { Limiting } \\
\text { options }\end{array}$ & $\begin{array}{l}\text { "I'm just too slow at getting everything else done, and I think I just kind } \\
\text { of cut back on everything that I want to do. The hobbies, exercises, and } \\
\text { socializing get cut first." }\end{array}$ \\
\hline & $\begin{array}{l}\text { Changes in } \\
\text { social roles }\end{array}$ & $\begin{array}{l}\text { "Before my stroke, I was a real estate sales lady. I had my own car, I } \\
\text { could drive. After the stroke, I had to give up my car, give up driving, } \\
\text { give up my job. Now I hardly do anything." }\end{array}$ \\
\hline & & $\begin{array}{l}\text { "I think it's actually easier now that I'm not working to eat healthy. I } \\
\text { think work was more of a stressor. I made the wrong choice a little more. } \\
\text { I think my friends have less influence than they did." }\end{array}$ \\
\hline \multirow[t]{3}{*}{$\begin{array}{l}\text { Environmental } \\
\text { Forces }\end{array}$} & $\begin{array}{l}\text { Formal } \\
\text { caregivers }\end{array}$ & $\begin{array}{l}\text { "And the doctor said, if you change your diet, go vegan, and eliminate } \\
\text { oils, you would probably be able to get off of all the medication. So I } \\
\text { converted to veganism in the hospital." }\end{array}$ \\
\hline & & $\begin{array}{l}\text { "My doctor told me that no matter what I did, I'd be on medication for } \\
\text { all my life. And I refused to accept that and take the medications. As } \\
\text { long as I do the exercises and I eat right, my blood pressure will go } \\
\text { down." }\end{array}$ \\
\hline & $\begin{array}{l}\text { Informal } \\
\text { caregivers }\end{array}$ & $\begin{array}{l}\text { "I started coming to this exercise class. Sometimes I don't feel like } \\
\text { coming but my daughters drag me, and then I have to come. I feel 100\% } \\
\text { better." }\end{array}$ \\
\hline
\end{tabular}

"Well, my kids, they always seem a little surprised at whatever I do [...] They sometimes do too much for me."

Seeking "I think I've learned more about health since I've had my stroke than information ever before in my life because I think that you can't beat it unless you understand it."

Re-evaluation: $\quad$ Priorities and My eating habits have substantially changed for the worse. And it's priorities and standards whatever is most convenient, easy for me to do. [...]. Basically, if it's attributions anything more than a TV dinner, I am at a complete loss."

"I don't mean to sound like a snob, but my standards are much higher now. I used to think it was okay to eat greasy, fried foods, and now I wouldn't touch it [...].",

Stroke cause "I don't know exactly all the problems that cause a stroke, but I know that my sugar and sodium intake contributed to it. [...] So I had to start learning how to eat properly, and that's my goal."

"According to what I've read, the lack of exercise sometimes is a factor of diabetes, and it's also a factor of having a stroke. So if I want to avoid that pitfall in the future, it's incumbent upon me to keep myself in the best physical condition that I can [...]." 


\begin{tabular}{|c|c|c|}
\hline & & $\begin{array}{l}\text { what you set out to do, you start feeling like you are making some } \\
\text { progress, you feel more confident, and you feel better about yourself." }\end{array}$ \\
\hline \multirow{6}{*}{$\begin{array}{l}\text { Resiliency: } \\
\text { finding } \\
\text { motivation and } \\
\text { solutions }\end{array}$} & $\begin{array}{l}\text { Self- } \\
\text { determination }\end{array}$ & $\begin{array}{l}\text { "Well, the thing in which - that keeps me on track is my desire to get } \\
\text { better. I want to get back to doing what I used to do. And, I believe I can } \\
\text { do it, but I just have to be cautious and persistent about doing it." }\end{array}$ \\
\hline & & $\begin{array}{l}\text { "I just refuse to accept restrictions, and I've been that way my whole life. } \\
\text { I've never accepted no." }\end{array}$ \\
\hline & $\begin{array}{l}\text { Environmental } \\
\text { planning }\end{array}$ & "I only take nutritious food into the house now." \\
\hline & & $\begin{array}{l}\text { "I just like to make sure that there's fruit and vegetables in the house and } \\
\text { that I have access to them. And I - I've been trying to eat those rather } \\
\text { than eating junk food, which I've been trying not to keep in the house." }\end{array}$ \\
\hline & $\begin{array}{l}\text { Outcome } \\
\text { expectations }\end{array}$ & $\begin{array}{l}\text { "I'm not exactly where I want to be - everything has not returned to my } \\
\text { left side yet, but exercising and eating right and watching my weight is } \\
\text { getting me closer." }\end{array}$ \\
\hline & & $\begin{array}{l}\text { "Getting enough sleep and exercising are the things that contribute to me } \\
\text { being able to mentally be stronger and help resist stress or negativity." }\end{array}$ \\
\hline \multirow[t]{4}{*}{$\begin{array}{l}\text { Negative } \\
\text { Affectivity }\end{array}$} & $\begin{array}{l}\text { Stress and } \\
\text { anxiety }\end{array}$ & $\begin{array}{l}\text { "About the first three, man, it was fear. I couldn't sleep. After having the } \\
\text { stroke, I was so worried about having another one." }\end{array}$ \\
\hline & & $\begin{array}{l}\text { "I feel almost like a prisoner in my own house. And when you can't do } \\
\text { something and you want to do something, it makes you frustrated. [...] } \\
\text { And not being able to get out and about and do additional things like } \\
\text { exercise and go for a walk makes me more frustrated." }\end{array}$ \\
\hline & $\begin{array}{l}\text { Self- } \\
\text { consciousness }\end{array}$ & $\begin{array}{l}\text { "I just don't like going to the gym. I don't like it when other people } \\
\text { notice me struggling. They take pity on me, and I don't like that." }\end{array}$ \\
\hline & & $\begin{array}{l}\text { "It's just this and the effect the stroke had on me, and it's made me very } \\
\text { sensitive [about my body]. You know, I find myself questioning myself } \\
\text { on everything I do - whether I'm doing the right thing or whether I'm } \\
\text { doing it well enough." }\end{array}$ \\
\hline
\end{tabular}


949 Table 5: Integration of qualitative and quantitative data

\begin{tabular}{|c|c|c|c|}
\hline Approach & Meta-inference & Quantitative data & Qualitative data \\
\hline $\begin{array}{l}\text { Behavior } \\
\text { change } \\
\text { principles }\end{array}$ & $\begin{array}{l}\text { Reciprocal } \\
\text { determinism } \\
\text { correspondence }\end{array}$ & $\begin{array}{l}\text { Moderate correlations between } \\
\text { activities of daily living and } \\
\text { multiple healthy behaviors; i.e., } \\
\text { BMI }(\mathrm{r}=-0.49) \text {, physical activity } \\
(0.41) \text {, Sleep }(\mathrm{r}=-0.55) \text {, healthy } \\
\text { eating habits }(\mathrm{r}=0.42) \\
\text { Moderate correlation between } \\
\text { hand impairments and healthy } \\
\text { eating habits }(\mathrm{r}=0.45) \text { and non- } \\
\text { significant correlations between } \\
\text { hand impairments and physical } \\
\text { activity }(\mathrm{r}=0.20) \text {, sleep }(\mathrm{r}= \\
0.20) \text {, and BMI }(\mathrm{r}=0.05)\end{array}$ & $\begin{array}{l}\text { Descriptions of why } \\
\text { restrictions in activities of } \\
\text { daily living influence multiple } \\
\text { behaviors and differences in } \\
\text { how impairments influence } \\
\text { single or multiple behaviors } \\
\text { Descriptions of how mobility } \\
\text { impairments interact with } \\
\text { environmental factors to } \\
\text { restrict physical activity } \\
\text { options only or restrict } \\
\text { physical activity options and } \\
\text { healthy eating habits }\end{array}$ \\
\hline $\begin{array}{l}\text { Global } \\
\text { health / } \\
\text { behavioral } \\
\text { category }\end{array}$ & $\begin{array}{l}\text { Circumstantial } \\
\text { linkages }\end{array}$ & $\begin{array}{l}\text { Small non-significant correlations } \\
\text { between physical activity and } \\
\text { nutrition }(\mathrm{r}=0.33) \text { and between } \\
\text { physical activity and mobility ( } \mathrm{r}= \\
0.39)\end{array}$ & $\begin{array}{l}\text { Descriptions of the linkages } \\
\text { between physical activity and } \\
\text { nutrition based on the } \\
\text { circumstances of the } \\
\text { participants } \\
\text { Perceptions of how behaviors } \\
\text { are prioritized and how traits } \\
\text { may influence behaviors, such } \\
\text { as how resiliency facilitates } \\
\text { multiple behaviors and how } \\
\text { negative affectivity hinders } \\
\text { multiple behaviors }\end{array}$ \\
\hline $\begin{array}{l}\text { Association } \\
\text { between } \\
\text { behaviors }\end{array}$ & $\begin{array}{l}\text { Sleep } \\
\text { disturbance } \\
\text { ripple effect }\end{array}$ & $\begin{array}{l}\text { Moderate correlations between } \\
\text { sleep and multiple healthy } \\
\text { behaviors; BMI }(\mathrm{r}=0.48), \\
\text { physical activity }(\mathrm{r}=-0.48) \text {, and } \\
\text { ADL limitations }(\mathrm{r}=-0.55)\end{array}$ & $\begin{array}{l}\text { Descriptions of how sleep } \\
\text { disturbances decreased } \\
\text { motivation to engage in } \\
\text { multiple healthy behaviors }\end{array}$ \\
\hline
\end{tabular}


950 Figure 1: Conceptual model for engaging in multiple healthy behaviors among Individuals with 951 stroke

\section{Impairments (QUAL and QUAN)}

Fatigue and pain

Lower-extremity mobility Activities of daily living
Implications and Consequences (QUAL)

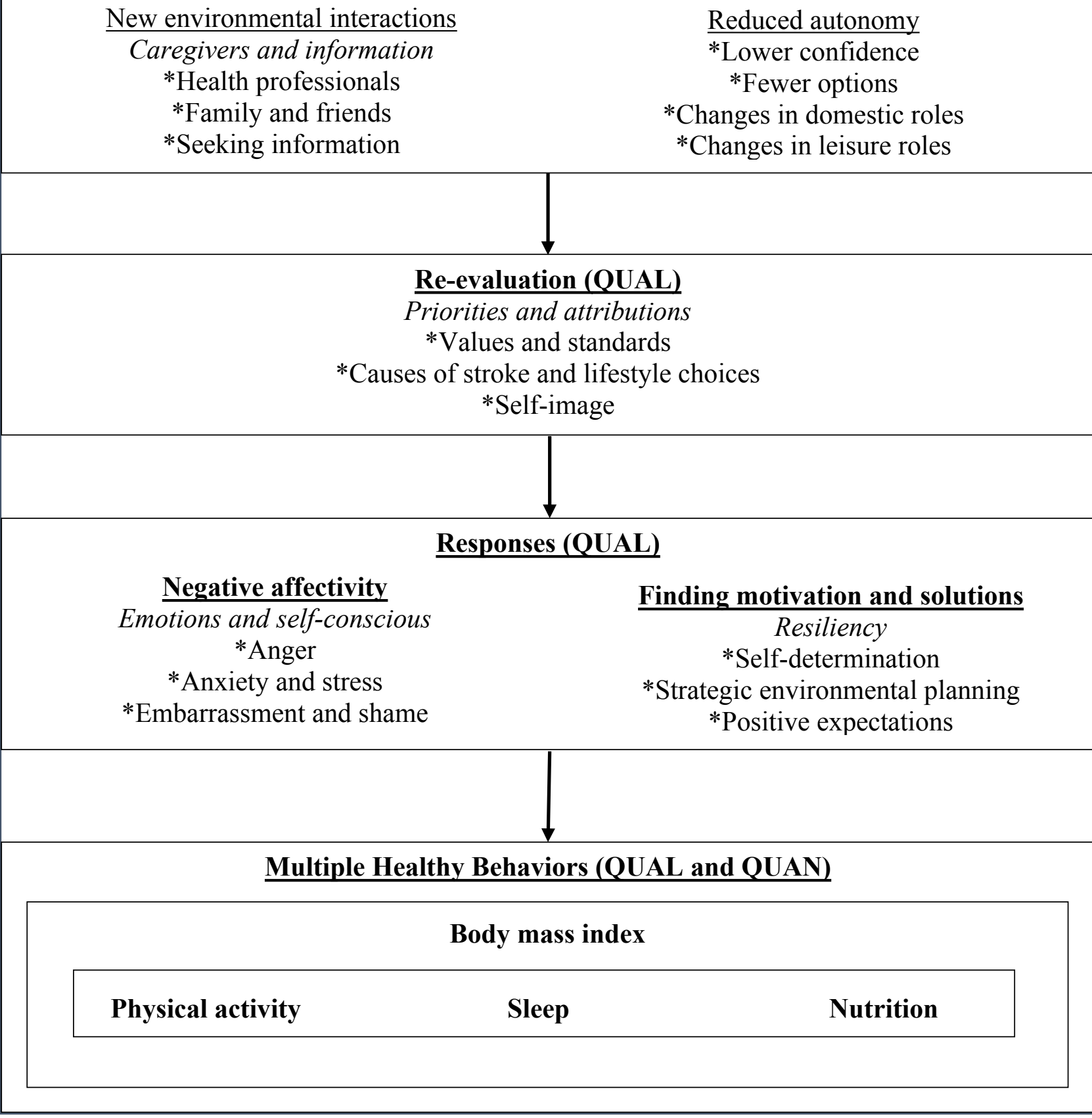


953 Note: QUAL = derived from qualitative data; QUAN = derived from quantitative data 